\title{
INTEGRAL OBSERVATIONS OF THE TRANSIENT SOURCE IGR J19140+098
}

C. Cabanac, ${ }^{1}$ J. Rodriguez, ${ }^{3,2}$ P. O. Petruc ${ }^{1}{ }^{1}$ G. Henri, ${ }^{1}$ D. C. Hamnikainen, ${ }^{4}$ and P. Durouchonx ${ }^{3}$

IGR J19140+098 was (re-)discovered during the early INTEGRAL observations of GRS1915+105 in March 2003. The following observations by INTEGRAL and RXTE show significant variability on various time scales from $100 \mathrm{~s}$ to $1 \mathrm{ks}$, but no pulsations. The ISGRI spectra show strong spectral variability in the 20-80keV range. Combined JEMX_2ISGRI spectra are well fitted by a "multicoulour" disk model with a rather high inner disk temperature $(\simeq 1.8 \mathrm{keV})$. RXTE data also reveal an apparently broad ionised iron line not detected in earlier INTEGRAL observations. These results are compatible with a galactic X-ray binary source.

IGR J19140+098 (Simbad corrected name IGR J19140+0951) was observed in the FOV of IBIS, the imager aboard INTEGRAL, for the first time during an observation of GRS1915+105 (PI : Hannikainen) on 6th March 2003 ( Spacecraft revolution \#48, 88 ks). The source is about 1' close to GRS1915+105 and has been observed after in two other revolutions: Rev \#69 (resp \#70) 9th May (resp 10th11th May) during $76 \mathrm{ks}$ (resp $96 \mathrm{ks}$ ) but not in Rev \#59 on 9th April (90 ks). RXTE observed the source on March 10th 2003 during 3 ks (public data). We show hereafter the resulting images, spectra and lightcurves using ISGRI (first layer detector of IBIS instrument, energy range : $20 \mathrm{keV}-10 \mathrm{MeV}$ ) and JEMX_2 (energy-range 3-21 keV).

The source seems to be rather variable on various time scales: it varies on a monthly timescale. The ISGRI light curves show that the flux can increase by a factor of 3 in 2000s. RXTE light curves (16 $\mathrm{s}$ bin size) seem to define a minimum variation time scale. The autocorrelation function indicates a typical correlation time of about $150 \mathrm{~s}$. Using the causality argument, it corresponds to a size of about 3 AU. No QPOs were identified in the PDS The integrated ISGRI flux in the $13-100 \mathrm{keV}$ energy range varied from $1.810^{-9} \mathrm{erg}^{-1} \mathrm{~cm}^{-2}$ (highest value observed) in rev\#70 to the minimum de-

\footnotetext{
${ }^{1}$ Laboratoire d'Astrophysique de Grenoble, France

${ }^{2}$ ISDC, Versoix, Switzerland.

${ }^{3} \mathrm{CEA}$, Saclay, France.

${ }^{4}$ Observatory, University of Helsinki, Finland.
}

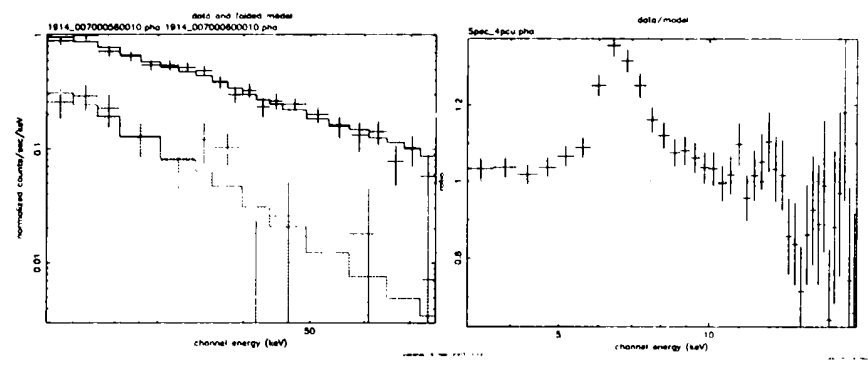

Fig. 1. Left 20-80 keV ISGRI spectra of the source during rev\#70 separated by $8.6 \mathrm{ks}$ : Resulting photon index are 2.210 .15 (black) and 3.81.0 (red). Right Ratiu botworll the RXTE-PCA data and the model chosen (simple powerlaw with $N_{H}$ fixed to $5.610^{22} \mathrm{~cm}^{-2}$ ).

tection level in rev\# 59. JF,MX_2 and ISGRI spectra show that the emission can be modelized consist enttly by a "hard" power law in the $10-100 \mathrm{keV}$ energy range $(\Gamma=1.7)$. Adding a disk model for the lower energy emission (freezing the power law paramu(crs) improve the fits (then the temperature obtained is rather high $T_{i n} \simeq 1.8 \mathrm{keV}$ ). Above $20 \mathrm{keV}$ and under $80 \mathrm{keV}$, spectra are well fitted $\mathrm{l} y$ a simple power law model but show strong spectral variability (Fig 1). Adding a gaussian line to JEMX_2-ISGRI spectra does not reduce the ${ }^{2}$ significantly whereas it does in RXTE data, performed two days lial'r. The line obtained is then consistent, with a broad gallssian line $\sigma=0.4 \mathrm{keV}$ ) (contered on $6.7 \mathrm{kr} V$, compatible with a ionized Fe $K_{\alpha}$ line (Fig. 1). The source IGR J19140+0951 has been re-discovered by INTEGRAL. The spectra are well-fitted by a power-law + multicolour disk. A broad and apparently ionized iron line is clearly detected in RXTE public dati but not in INTEGRAL observations taken 2 davs earlier. The source exhibits strong spertral and flux variabilities more typical of an X-ray binary than of an AGN. This source looks like IGR 16332()-4751 another X-ray source (re-) discovered by INTEGR AL which manifest similar variable behaviour (Rodriguez $e_{t}^{t}$ al. 2003), and which is most probably a HALAB hosting a galactic neutron star.

\section{REFERE.NCES}

Hannikainen et al. 2003, [astro-ph/309532]

Hannikairen, Rodriguez \& Potschmidt 2003. I.tl' C' S1: Rodriguez et, al. 2003, A\&A, 407, 41-45

Swank \& Mirkwardt, 2003, ATEL \#128 